\title{
Muscular atrophy of caveolin-3-deficient mice is rescued by myostatin inhibition
}

\author{
Yutaka Ohsawa, ${ }^{1}$ Hiroki Hagiwara, ${ }^{1}$ Masashi Nakatani, ${ }^{2}$ Akihiro Yasue, ${ }^{3}$ Keiji Moriyama, ${ }^{3}$ \\ Tatsufumi Murakami, ${ }^{1}$ Kunihiro Tsuchida, ${ }^{2}$ Sumihare Noji, ${ }^{4}$ and Yoshihide Sunada1
}

\begin{abstract}
1Division of Neurology, Department of Internal Medicine, Kawasaki Medical School, Kurashiki, Japan. 2Division for Therapies against Intractable Diseases, Institute for Comprehensive Medical Science, Fujita Health University, Toyoake, Japan. ${ }^{3}$ Department of Orthodontics, Faculty of Dentistry, and ${ }^{4}$ Department of Biological Science and Technology, Faculty of Engineering, The University of Tokushima, Tokushima, Japan.
\end{abstract}

\begin{abstract}
Caveolin-3, the muscle-specific isoform of caveolins, plays important roles in signal transduction. Dominant-negative mutations of the caveolin-3 gene cause autosomal dominant limb-girdle muscular dystrophy 1C (LGMD1C) with loss of caveolin-3. However, identification of the precise molecular mechanism leading to muscular atrophy in caveolin-3-deficient muscle has remained elusive. Myostatin, a member of the muscle-specific TGF- $\beta$ superfamily, negatively regulates skeletal muscle volume. Here we report that caveolin-3 inhibited myostatin signaling by suppressing activation of its type I receptor; this was followed by hypophosphorylation of an intracellular effector, Mad homolog 2 (Smad2), and decreased downstream transcriptional activity. Loss of caveolin-3 in P104L mutant caveolin-3 transgenic mice caused muscular atrophy with increase in phosphorylated Smad2 (p-Smad2) as well as p21 (also known as Cdkn1a), a myostatin target gene. Introduction of the myostatin prodomain, an inhibitor of myostatin, by genetic crossing or intraperitoneal administration of the soluble type II myostatin receptor, another inhibitor, ameliorated muscular atrophy of the mutant caveolin-3 transgenic mice with suppression of $\mathrm{p}-\mathrm{Smad} 2$ and $p 21$ levels. These findings suggest that caveolin-3 normally suppresses the myostatin-mediated signal, thereby preventing muscular atrophy, and that hyperactivation of myostatin signaling participates in the pathogenesis of muscular atrophy in a mouse model of LGMD1C. Myostatin inhibition may be a promising therapy for LGMD1C patients.
\end{abstract}

\section{Introduction}

Caveolins are 21- to 24-kDa integral membrane proteins and are principal components of flask-shaped invaginations of the plasma membrane known as caveolae. These proteins play important roles in signal transduction and vesicular trafficking (1-3). They directly bind to and regulate specific lipid and lipid-modified molecules including cholesterol, $G$ proteins, $G$ protein-coupled receptors, Src family protein kinases, Ha-Ras, and nitric oxide synthases $(1,2)$. The interaction between caveolin and lipid-modified proteins is mediated by a specific caveolin-binding motif on the target protein and by a scaffolding domain in caveolin (3). There are 3 mammalian caveolin genes, namely, caveolin-1, -2 , and -3 . Caveolin-1 and -2 are coexpressed and form heterooligomers in nonmuscle cells whereas caveolin-3 is muscle specific and forms homooligomers in muscle cells $(1,2)$.

Loss of caveolin-3 resulting from dominant-negative mutations of the caveolin-3 gene causes autosomal dominant limb-girdle muscular dystrophy 1C (LGMD1C) (4). We previously generated Tg mice overexpressing the Pro104Leu mutant caveolin-3 (CAV-3 ${ }^{\text {P104L }}$ ) as a model for LGMD1C (5). The skeletal muscle pathology of the $\mathrm{Tg}$ mice includes myopathy characterized by severe skeletal muscle atrophy and a deficiency in caveolin-3. We also found a significant increase in neuronal nitric oxide synthase activity in their skeletal muscle. Other groups have demonstrated mislocalization of Src

Nonstandard abbreviations used: ActRIIB, activin receptor IIB; ALK4, activin receptor-like kinase 4; Cav3 ${ }^{\mathrm{P} 104 \mathrm{~L}}$ mice, $\mathrm{Tg}$ mice overexpressing the P104L mutant caveolin-3; CDK, cyclin-dependent kinase; LGMD1C, limb-girdle muscular dystrophy $1 \mathrm{C} ; \mathrm{Mstn}^{\text {Pro }}$ mice, $\mathrm{Tg}$ mice overexpressing the myostatin prodomain in skeletal muscle; p-, phosphorylated; Smad2, Mad homolog 2; TA, tibialis anterior.

Conflict of interest: The authors have declared that no conflict of interest exists. Citation for this article: J. Clin. Invest. 116:2924-2934 (2006). doi:10.1172/JCI28520. and dysferlin to the Golgi apparatus $(6,7)$. Despite these findings, the precise molecular mechanism leading to muscular atrophy in caveolin-3-deficient skeletal muscle remains to be elucidated.

Myostatin is a member of the TGF- $\beta$ superfamily and plays an essential role in the negative regulation of skeletal muscle volume (8). Overexpression of myostatin causes severe muscular atrophy $(9,10)$ whereas targeted disruption of myostatin markedly increases muscle mass in mice $(8,11)$. We also generated $\mathrm{Tg}$ mice overexpressing the myostatin prodomain in skeletal muscle $\left(\mathrm{Mstn}^{\mathrm{Pro}}\right)(12)$, an inhibitor of myostatin activation (13-17). Like other TGF- $\beta$ superfamily members (18), myostatin is synthesized as a precursor protein and undergoes proteolytic processing to generate an $\mathrm{N}$ terminal prodomain and a biologically active, $C$ terminal disulfide-linked dimer (19). In the inactive state, the prodomain binds to the $\mathrm{C}$ terminal myostatin dimer and strongly inhibits its biological activity (13). The circulating active form of myostatin directly binds to and activates activin receptor IIB (ActRIIB), a type II serine/threonine kinase receptor (19). This, in turn, activates the type I serine/threonine kinase receptor activin receptor-like kinase 4 (ALK4) or ALK5 at the plasma membrane $(20,21)$. The activation of a heteromeric receptor complex consisting of type II and type I serine/threonine kinase receptors induces the phosphorylation of intracellular effectors Mad homolog 2 $(\operatorname{Smad} 2)$ and Smad3 $(20,21)$. Phosphorylated Smad2 and Smad3 translocate from the cytoplasm to the nucleus, where they regulate the transcription of specific target genes (20-22).

Recently, caveolin-1 was reported to inhibit the activation of the type I receptor for TGF- $\beta 1$, which induces growth arrest in nonmuscle cells (23). Upon consideration of molecular analogy and tissue distribution, we hypothesized that caveolin-3 inhibits myostatin signaling in muscle cells in a similar manner. Accordingly, an increase in myostatin activity resulting from loss of 
A
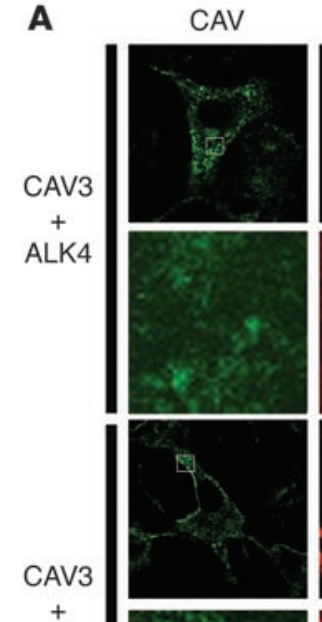

ALK5
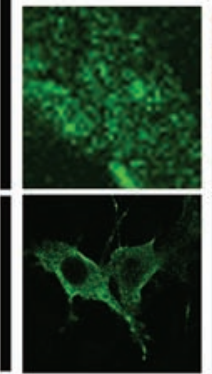

CAV-3

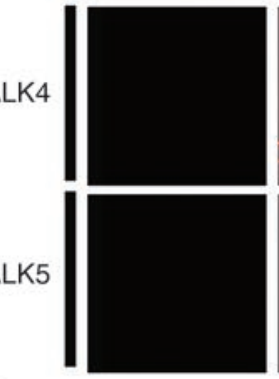

C
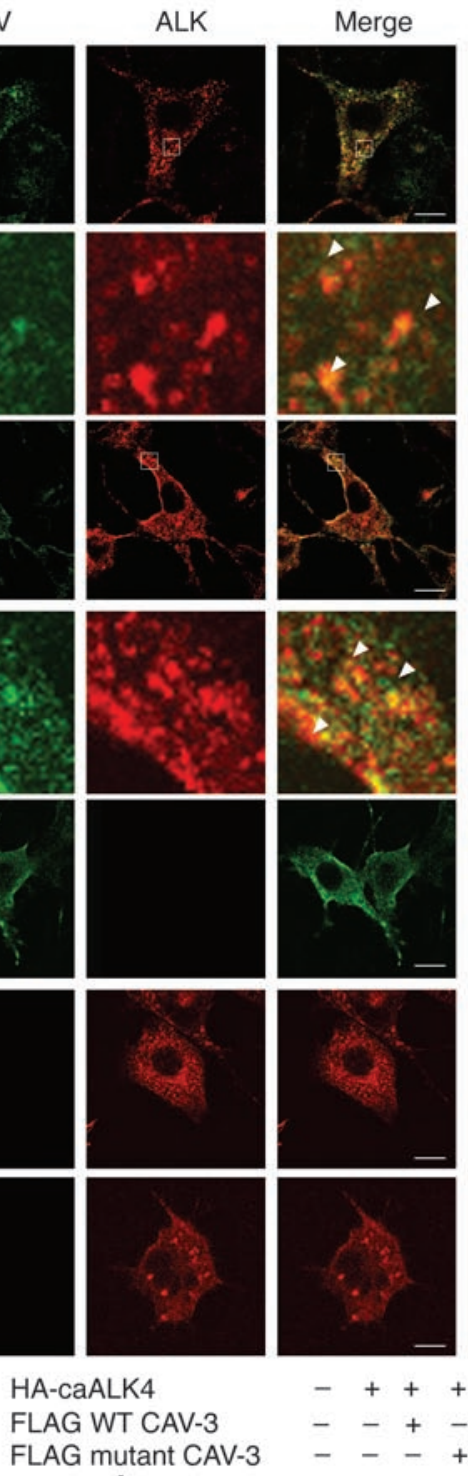

FLAG mutant CAV-3 $\quad-\quad-\quad+$

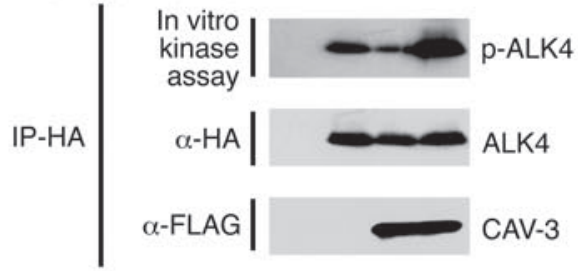

B
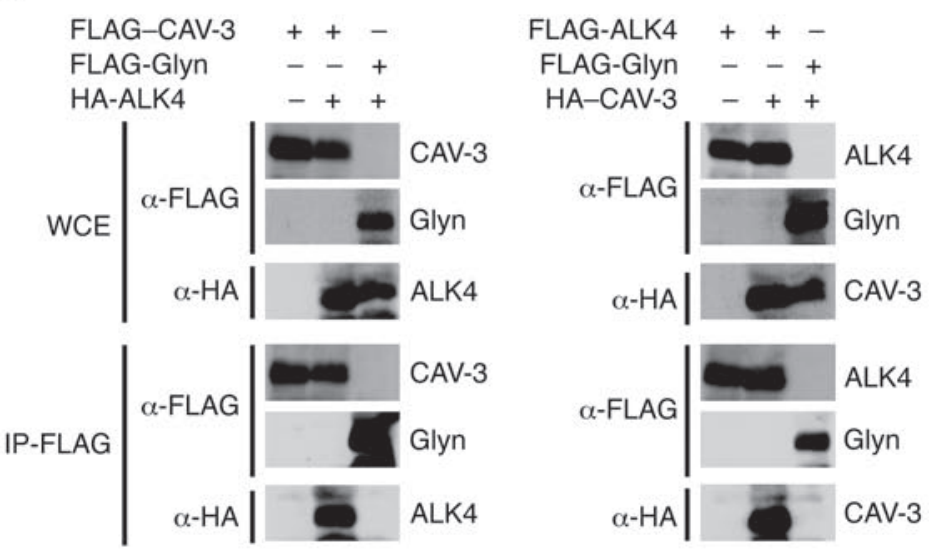
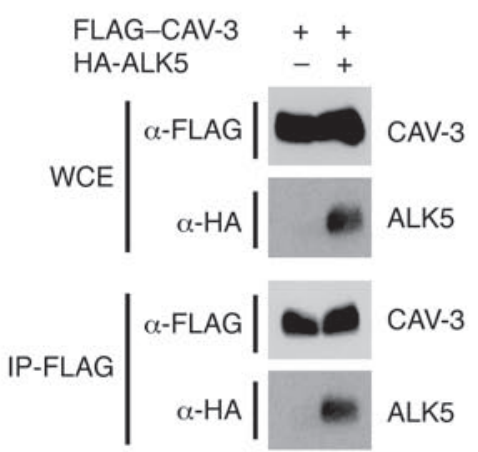

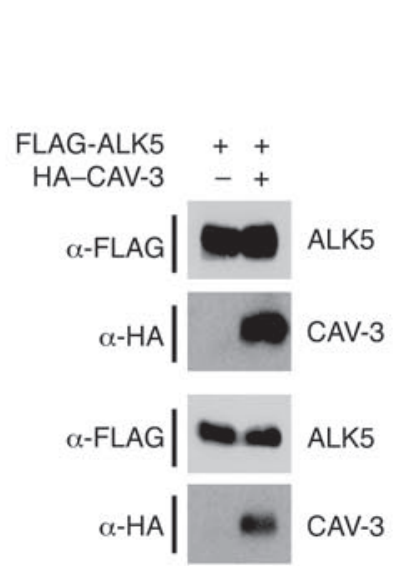

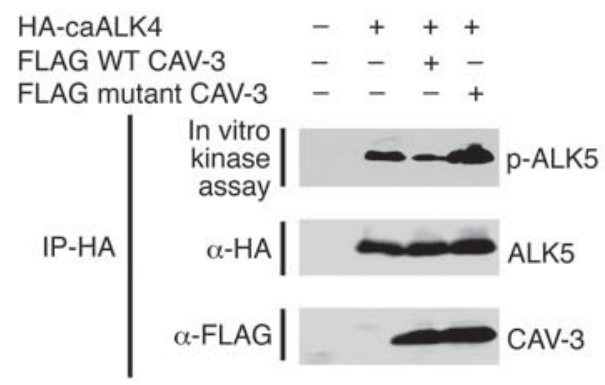

Figure 1

Caveolin-3 binds to the type I myostatin receptors and suppresses their activation. (A) Colocalization of caveolin-3 (CAV-3) and type I myostatin receptors (ALK4 and ALK5). COS-7 cells transfected with caveolin-3 and/or ALK4 or ALK5 were double labeled with an anti-rabbit caveolin-3 polyclonal $\mathrm{Ab}(\mathrm{CAV})$ and an anti-mouse HA mAb (ALK). The enlarged images of the boxed regions are shown below the original pictures. White arrowheads indicate colocalization of caveolin-3 with ALK4 or ALK5. Scale bars: $10 \mu \mathrm{m}$. (B) Interaction of caveolin-3 and ALK4 (upper panels) or ALK5 (lower panels). Cell lysates from COS-7 cells cotransfected with FLAG- or HA-tagged caveolin-3 (CAV-3) and either HA- or FLAG-tagged type I receptor were immunoprecipitated with anti-FLAG agarose gel, then immunoblotted using anti-FLAG mAb $(\alpha-F L A G)$ or anti-HA mAb $(\alpha-H A)$. Also, whole cell extracts (WCE) were analyzed by immunoblotting with the same Abs. Glycogenin (Glyn) was used as a negative control for immunoprecipitation. (C) In vitro autophosphorylation of constitutively active ALK4 and ALK5. Cell lysates from COS-7 cells cotransfected with FLAG-tagged wild-type or P104L mutant caveolin-3 and HA-tagged constitutively active ALK4 (HA-caALK4) or ALK5 (HA-caALK5) were immunoprecipitated with anti-HA agarose. The in vitro kinase reaction was initiated by the addition of kinase reaction buffer and [ $\left.\gamma-{ }^{32} \mathrm{P}\right] \mathrm{ATP}$. Phosphorylated caALK4 or caALK5 was detected by autoradiography. Immunoprecipitated caALK4, caALK5, and caveolin-3 were analyzed by immunoblotting with anti-HA mAb $(\alpha-\mathrm{HA})$ or anti-FLAG $(\alpha-F L A G)$ mAb. Bands corresponding to phosphorylated type I receptor (in vitro kinase assay), total type I receptor $(\alpha-H A)$, and caveolin-3 ( $\alpha-F L A G)$ are shown. 
A

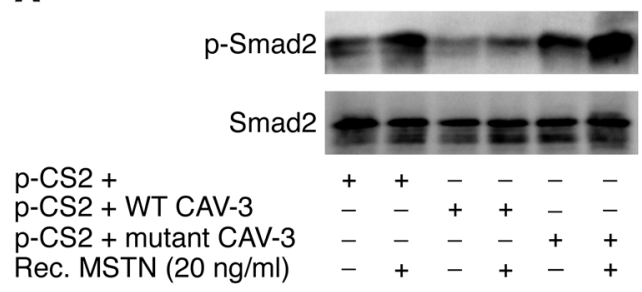

B

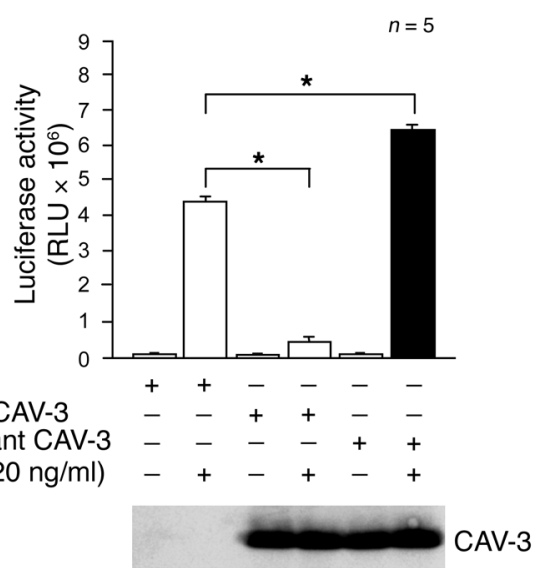

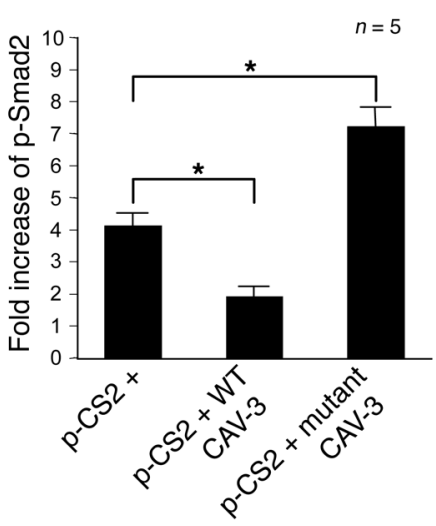

Figure 2

Caveolin-3 inhibits intracellular myostatin (MSTN) signaling in vitro. (A) Immunoblot analysis of total Smad2 (Smad2) and p-Smad2 in cell lysates from COS-7 cells transfected with empty vector ( $\mathrm{p}-\mathrm{CS} 2+$ ), wild-type caveolin-3 (p-CS2 + wild-type CAV-3), or P104L mutant caveolin-3 (p-CS2 + mutant CAV-3) in the presence or absence of recombinant myostatin (rec. MSTN) (left). Quantification of the fold increase of phosphorylated Smad2 in cell lysates in response to myostatin (right). Data are expressed as mean \pm SD $(n=5)$. ${ }^{\star} P<0.05$. (B) Myostatin-induced luciferase activity in HEK293 cells cotransfected with a Smad-responsive reporter gene and empty vector, wild-type caveolin-3, or mutant caveolin-3. Data are expressed as mean $\pm \operatorname{SD}(n=5)$. ${ }^{*} P<0.05$. Immunoblot analysis of caveolin-3 in cell lysates is shown in the lower panel. caveolin-3 in muscle might participate in the pathogenesis of skeletal muscle atrophy in LGMD1C patients. Our caveolin-3deficient mouse model is a useful tool for investigating how myostatin signaling is altered in LGMD1C patients. In the current study, we investigated our hypothesis by examining the interaction between caveolin-3 and type I myostatin receptor signaling in vitro. Additionally, we generated and characterized the double-Tg mice (CAV-3 $\left.3^{\text {P104L }} / \mathrm{MSTN}^{\text {Pro }}\right)$ by heterozygous mating of the mutant caveolin-3-Tg mice (CAV-3 $\left.{ }^{\text {P104L }}\right)(5)$ with the mutant myostatin-Tg mice (MSTN ${ }^{\text {Pro }}$ ) overexpressing the prodomain myostatin (12) to investigate the effect of myostatin inhibition in vivo on caveolin-3-deficient muscular atrophy. Furthermore, we injected a soluble form of type II myostatin receptor, another myostatin inhibitor $(19,24)$, into the mutant caveolin-3-Tg mice to investigate the therapeutic potential of myostatin inhibition for the future treatment of LGMD1C patients.

\section{Results}

Caveolin-3 can associate with the type I myostatin receptor. We first investigated whether caveolin-3 can associate with the type I myostatin receptor in vitro. COS-7 monkey kidney cells cotransfected with FLAG-tagged caveolin-3 and HA-tagged type I receptor (ALK4 or ALK5) were double immunostained with an anti-rabbit caveolin-3 polyclonal $\mathrm{Ab}$ and an anti-mouse $\mathrm{mAb}$ against HA. Confocal microscopy showed that caveolin-3 colocalized with ALK4 or ALK5 (Figure 1A). We next investigated the potential interaction of caveolin-3 with the type I myostatin receptor. COS-7 cells cotransfected with FLAG- or HA-tagged caveolin-3 and HA- or FLAG-tagged type I receptor were lysed and precipitated with anti-FLAG agarose. We detected coprecipitation of caveolin-3 with type I receptors and, conversely, type I receptors with caveolin-3 by immunoblotting with an anti-HA Ab (Figure 1B). These results indicate that caveolin-3 associates with type I myostatin receptors.
Caveolin-3 negatively regulates activation of the type I myostatin receptor. The association of caveolin-3 with type I myostatin receptor raised the intriguing possibility that caveolin-3 regulates the serine/threonine kinase activity of ALK4 or ALK5. Therefore, to determine whether caveolin-3 can affect the phosphorylation status of type I myostatin receptors, we investigated the autophosphorylation of constitutively active ALK4 or ALK5 (Figure 1C). COS-7 cells were cotransfected with HA-tagged constitutively active type I receptor and FLAG-tagged wild-type or P104L mutant caveolin-3. Constitutively active type I receptor was immunoprecipitated with anti-HA agarose and subsequently subjected to an in vitro kinase reaction. We found that phosphorylation of ALK4 or ALK5 was decreased by the wild-type caveolin-3, and conversely, increased by the P104L mutant caveolin-3 (Figure 1C). These results indicate that caveolin-3 inhibits activation of the type I myostatin receptor whereas the P104L mutant caveolin-3 enhances activation of the type I myostatin receptor in vitro.

Caveolin-3 inhibits myostatin-induced phosphorylation of Smad2. Because the activation of type I receptor induces phosphorylation of an intracellular effector, $\operatorname{Smad} 2(20,21)$, we investigated whether the level of Smad2 phosphorylation is affected by caveolin-3. COS-7 cells transfected with either the wild-type or the P104L mutant caveolin-3 were stimulated with recombinant myostatin. Cell lysates were then subjected to immunoblot analysis using an $\mathrm{Ab}$ against $\mathrm{Smad} 2$ or phosphorylated Smad2 ( $\mathrm{p}$-Smad2). The amount of total Smad2 proteins was invariable (Figure 2A). The basal and fold increases of p-Smad2 levels were reduced in the wild-type CAV-3 transformant, but they were increased in the mutant CAV-3 transformant (Figure 2A). Thus, myostatin-induced Smad 2 phosphorylation was inhibited by the wild-type caveolin-3 and, conversely, enhanced by the mutant caveolin-3, indicating that caveolin-3 regulates intracellular myostatin signaling at the effector level. 
A

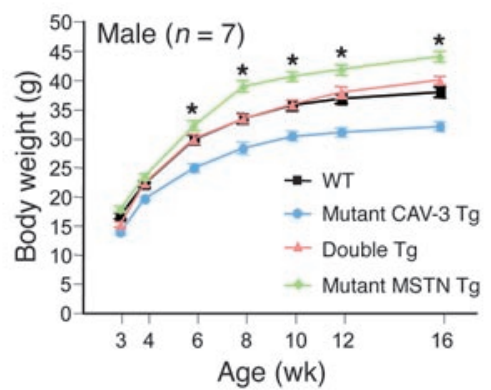

B

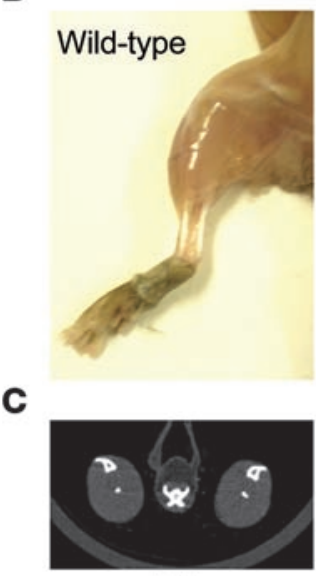

WT
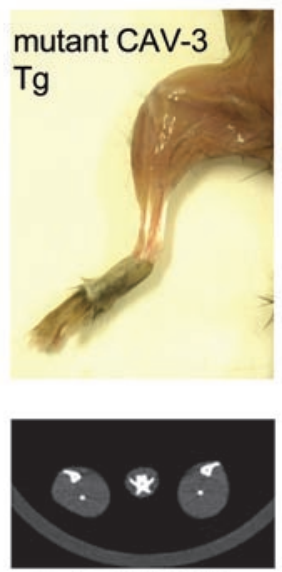

Mutant CAV-3 Tg
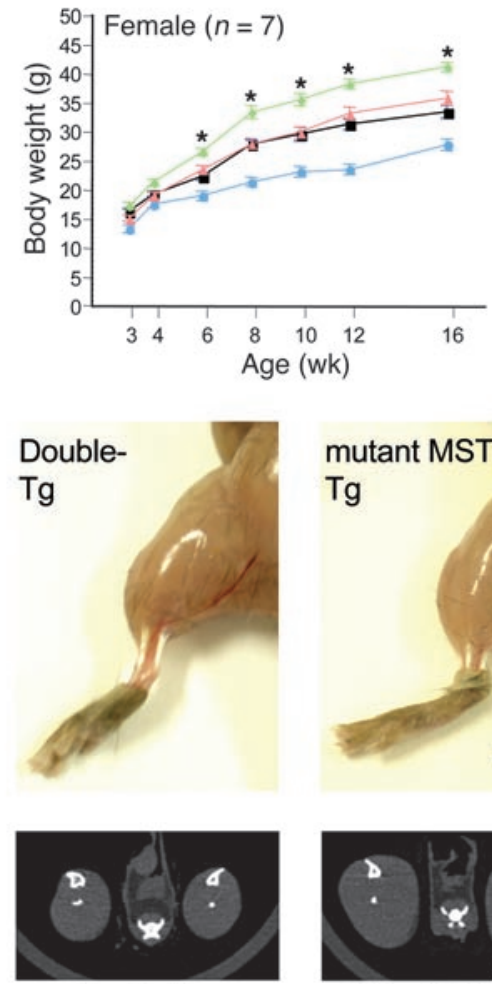

Double $\mathrm{Tg}$
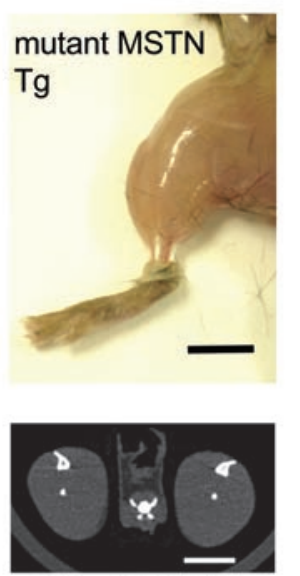

Mutant MSTN Tg

\section{Figure 3}

Myostatin inhibition reverses muscular atrophy of mutant caveolin-3-Tg mice. (A) Comparison of body weight of mice at $3,4,6,8,10,12$, and 16 weeks of age. Data are expressed as mean $\pm \mathrm{SD}(n=7) .{ }^{*} P<0.05$. (B) Appearance of skinned hind limbs from 10-week-old mice of the 4 distinct genotypes. Scale bar: $5 \mathrm{~mm}$. (C) Axial computed tomographic scans of the center of distal hind limbs in 10-week-old mice. Scale bar: 5 mm. Mutant CAV-3 Tg, CAV-3 $3^{\text {P104L; }}$ mutant MSTN Tg, MSTNPro; double Tg, CAV-3 ${ }^{\text {P104L/MSTNPro. }}$
Caveolin-3 inbibits myostatin-induced transcriptional activity. To determine the effect of caveolin-3 on myostatin-induced transcriptional activity, we performed a transcriptional assay using a Smad-responsive luciferase reporter gene $(13,25)$. HEK293 human embryonic kidney cells, which do not express endogenous caveolin-3 (Figure 2B), were cotransfected with the luciferase reporter and either empty vector, wild-type caveolin-3, or mutant caveolin-3. Stimulation with recombinant myostatin in HEK293 cells transfected with the empty vector caused a significant increase in luciferase activity above the basal level (Figure 2B). In the wildtype caveolin-3 transformant, myostatin-induced transcriptional activity decreased by approximately $90 \%$ (Figure $2 B$ ). In contrast, the mutant caveolin-3 transformant showed a 1.5 -fold increase in myostatin-induced transcriptional activity. These results indicate that wild-type caveolin-3 functionally inhibits myostatin signaling at the transcriptional level whereas P104L mutant caveolin-3 enhances myostatin signaling.

Introduction of the myostatin prodomain transgene reverses muscular atrophy in the mutant caveolin-3-Tg mice. To determine whether caveolin-3 regulates myostatin signaling in skeletal muscle in vivo and whether myostatin inhibition affects muscular atrophy in caveolin-3-deficient mice, we generated double-Tg mice showing both caveolin-3 deficiency and myostatin inhibition. Heterozygous mating of the mutant caveolin-3-Tg mice (5) with the mutant myostatin-Tg mice (12) gave rise to mice with 4 distinct genotypes: wild-type, mutant caveolin-3-Tg (CAV-3 $\left.3^{\text {P104L }}\right)$, mutant myostatin$\mathrm{Tg}\left(\mathrm{MSTN}^{\mathrm{Pro}}\right)$, and double Tg (CAV-3 $\left.{ }^{\text {P104L }} / \mathrm{MSTN}^{\text {Pro }}\right)$. The mRNA expression of the transgenes in skeletal muscle was confirmed by Northern blotting (Supplemental Figure 1; supplemental material available online with this article; doi:10.1172/JCI28520DS1). We examined the progressive changes in body weight between 3 and
16 weeks after birth (Figure 3A). The body weights for wild-type, mutant caveolin-3-Tg, and mutant myostatin-Tg were significantly different beginning at 6 weeks and until 16 weeks of age. The mutant caveolin-3-Tg mice were significantly smaller than wildtype mice whereas the double-Tg mice were significantly larger than the mutant caveolin-3-Tg mice and similar in size to the wildtype mice (Supplemental Figure 2). This tendency was found in both sexes. Examination of skinned hind-limb muscle showed that muscular atrophy in the mutant caveolin-3-Tg mice was reversed in the double-Tg mice (Figure 3B). This was also observed in axial computed tomographic scans (Figure 3C). These results suggest that inhibition of myostatin signaling with its prodomain reverses skeletal muscle atrophy in the mutant caveolin-3-Tg mice.

Myostatin inhibition reverses bypoplasia and bypotrophy in mutant caveolin-3-Tg mice. H\&E staining and laminin immunostaining of tibialis anterior (TA) muscle showed that reduced myofiber size in the mutant caveolin-3-Tg mice appeared to be reversed in the double-Tg mice even though they were still deficient in caveolin-3 (Figure 4A). To determine whether hypertrophy (i.e., increase of myofiber size) or hyperplasia (i.e., increase of myofiber number) contributed to the restoration of muscle mass in the double-Tg mice, we examined the distribution of single myofiber area and the total myofiber number in the TA muscle ( $n=5$; Figure 4 , B and C). Compared with the wild-type mice, the mutant caveolin-3-Tg mice showed a significant reduction in single myofiber area $(1984.7 \pm 660.2$ versus $844.8 \pm 320.2 \mu \mathrm{m}^{2} ; P<0.05$; Figure $4 \mathrm{~B}$ ) and total myofiber number $(1663.4 \pm 92.2$ versus $1125.2 \pm 68.6 ; P<0.05$; Figure $4 C)$. In contrast, the mutant myostatin- $\mathrm{Tg}$ mice exhibited a significant increase in single myofiber area $\left(2133.5 \pm 632.4 \mu \mathrm{m}^{2} ; P<0.05\right.$ versus wild-type; Figure 4B) and total myofiber number (3336.4 \pm 71.3 ; $P<0.05$ versus wild type; Figure 4C). In the double-Tg mice, both 
A

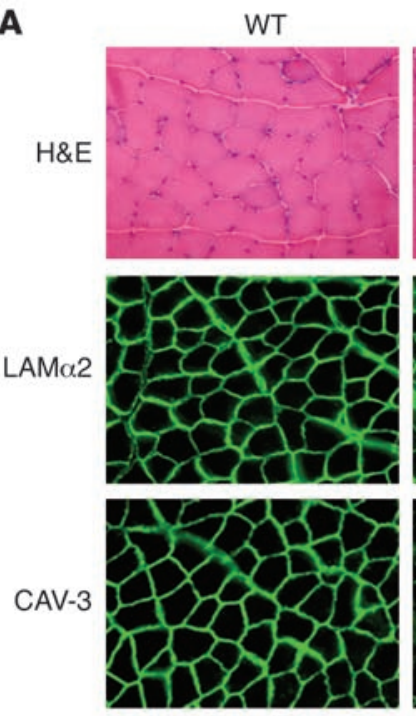

B
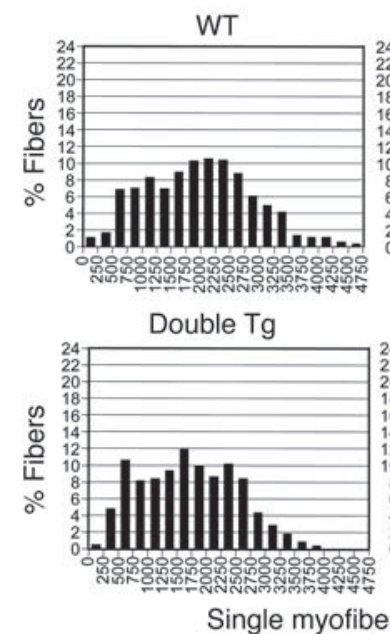

Mutant CAV-3 Tg
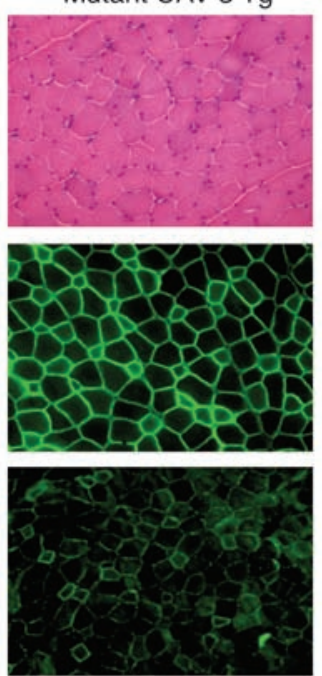

Mutant CAV-3 Tg

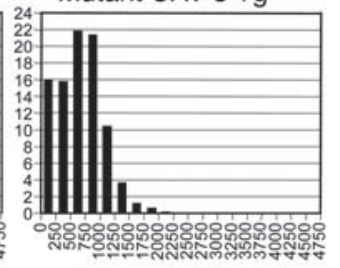

Mutant MSTN Tg

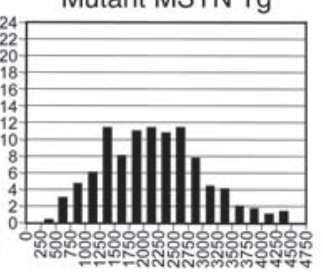

Double $\mathrm{Tg}$
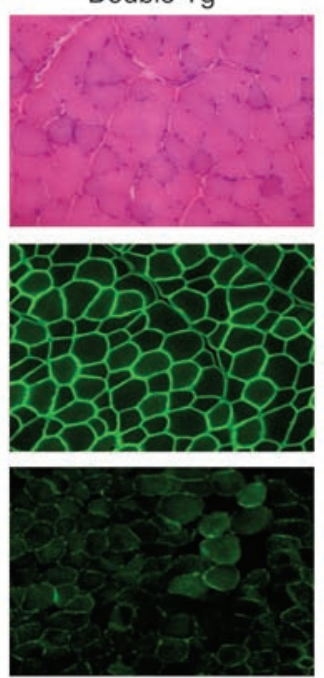

C

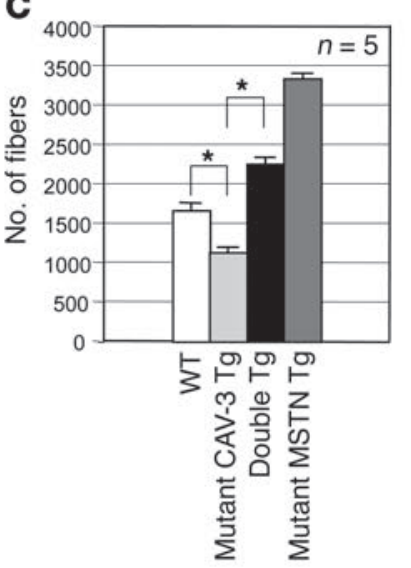

Mutant MSTN Tg
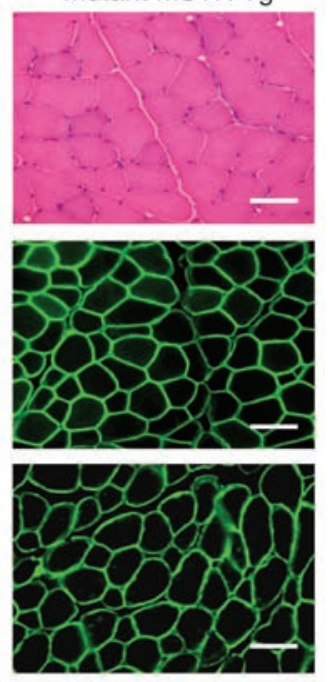

$n=5$

D

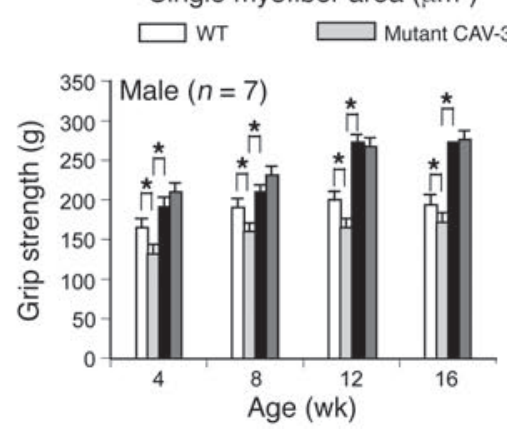

Tg Double $\mathrm{Tg}$

$\square$ Mutant MSTN Tg

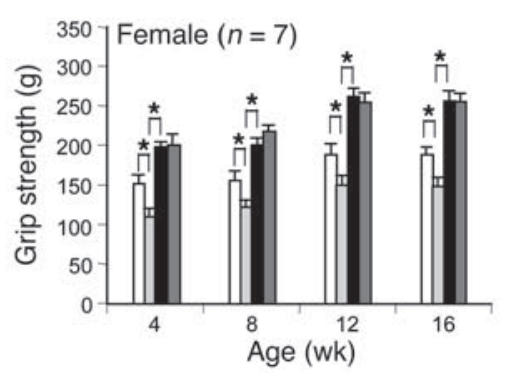

the single myofiber area $\left(1898.6 \pm 642.1 \mu \mathrm{m}^{2}\right.$; Figure $\left.4 \mathrm{~B}\right)$ and the total myofiber number $(2252.8 \pm 85.5$; Figure $4 \mathrm{C})$ were significantly $(P<0.05)$ increased compared with the mutant caveolin-3-Tg mice. Thus, myostatin inhibition reverses both myofiber hypotrophy and hypoplasia in caveolin-3-deficient skeletal muscle.

Myostatin inhibition reverses muscle weakness in the mutant caveolin-3-Tg mice in vivo. We evaluated skeletal muscle function by measuring the peak force (g) of grip strength between 4 and 16 weeks after birth. The mutant caveolin-3-Tg mice were significantly weaker, and the mutant myostatin-Tg mice were significantly stronger than the wild-type mice at all ages examined ( $n=7$; Figure 4D).
The double-Tg mice were also significantly stronger than the mutant caveolin-3-Tg mice and were as strong as the mutant myostatin-Tg mice (Figure 4D). Furthermore, a treadmill exercise test showed that impaired running ability in the mutant caveolin-3$\mathrm{Tg}$ mice was recovered in the double-Tg mice (Supplemental Figure 3). Muscle weakness in the caveolin-3-deficient mice was rescued by myostatin inhibition.

The amount of prodomain bound to endogenous myostatin is abundant in the mutant myostatin-Tg or the double-Tg mouse skeletal muscle. To examine the possibility that the transgene-derived prodomain binds to $\mathrm{C}$ terminal myostatin in vivo, we performed 
A

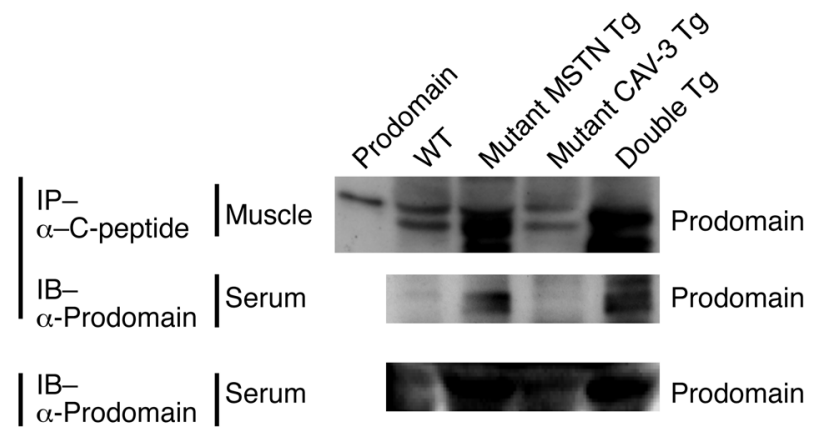

B

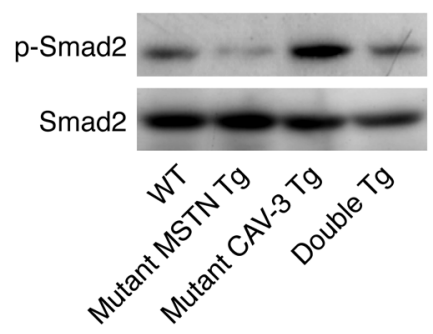

C

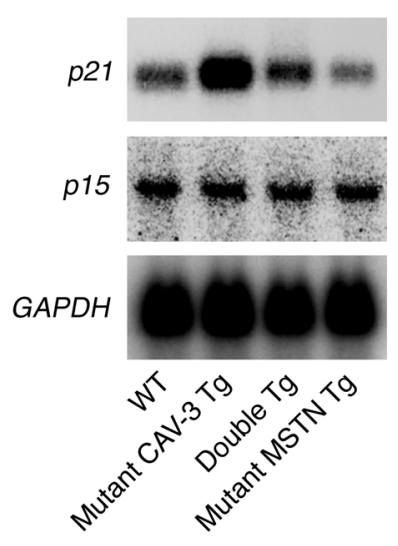

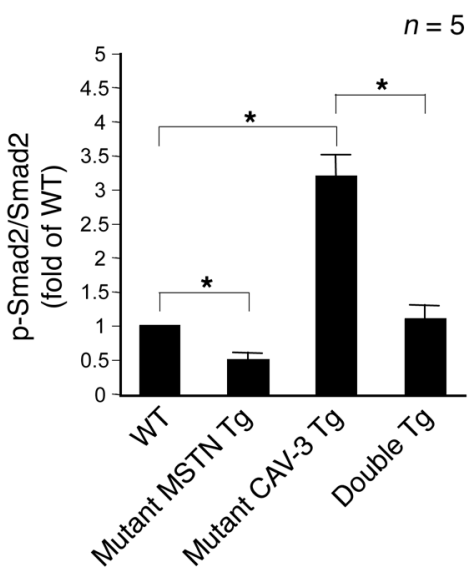

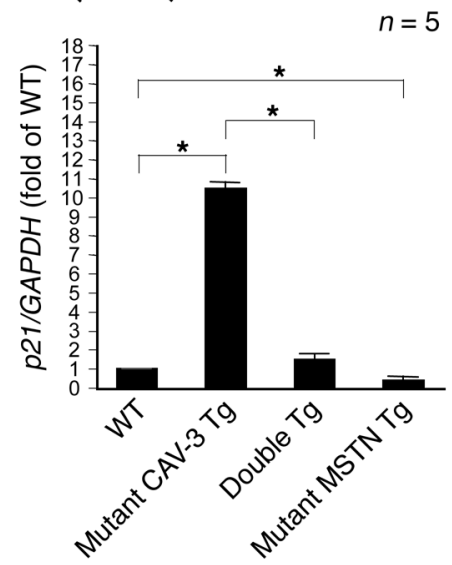

Figure 5

Myostatin inhibition suppresses hyperphosphorylation of Smad2 and increased $p 21$ expression in caveolin3-deficient muscle. (A) Prodomain C terminal myostatin complex was immunoprecipitated from mouse crude skeletal muscle homogenates (top panel) or sera (middle panel) with an $\mathrm{Ab}$ against the myostatin $\mathrm{C}$ terminal peptide ( $\alpha-C$-peptide), then immunoblotted (IB) using a polyclonal $A b$ against the prodomain ( $\alpha$-prodomain). Recombinant myostatin prodomain is used as a control. Immunoblot analysis of sera (bottom panel) shows an excessive amount of prodomain putatively derived from the transgene in the mutant myostatin-Tg mice as well as the double-Tg mice. (B) Immunoblot analysis of total Smad2 and p-Smad2 in crude skeletal muscle homogenates from mice (left). Quantification of the $\mathrm{p}$-Smad2/Smad2 ratio by densitometric analysis (right). Values are represented as fold increase with respect to wild-type mice. Data are expressed as mean $\pm \operatorname{SD}(n=5)$. ${ }^{*} P<0.05$. (C) Northern blot analysis in mouse skeletal muscle for $p 21$ and $p 15$, which are myostatin-dependent and -independent CDK inhibitors, respectively (left). Quantification of the $p 21 / G A P D H$ ratio by densitometric analysis (right). Values are represented as fold increase with respect to wild-type mice. Data are expressed as mean $\pm \mathrm{SD}(n=5)$. ${ }^{*} P<0.05$. immunoprecipitation analysis using an $\mathrm{Ab}$ against the $\mathrm{C}$ terminal myostatin, followed by immunoblotting with an $\mathrm{Ab}$ against the prodomain as described previously $(26,27)$. An excessive amount of prodomain was immunoprecipitated with the endogenous $\mathrm{C}$ terminal myostatin from the skeletal muscle and serum in both the mutant myostatin-Tg mice and the double-Tg mice (Figure 5A). The expression level of the prodomain was also very high in both mutant MSTN Tg and double-Tg mice in immunoblot analysis of sera (Figure 5A). These results suggest that transgene-derived prodomain binds to endogenous $\mathrm{C}$ terminal myostatin in vivo.

Myostatin inhibition suppresses increased Smad2 phosphorylation in caveolin-3-deficient atrophic muscle. To investigate the status of intracellular myostatin signaling in vivo, we examined the levels of Smad2 and p-Smad 2 in crude skeletal muscle homogenates ( $n=5$ per genotype) by immunoblotting and subsequent densitometric analysis (Figure 5B). The amount of total Smad2 protein was unchanged whereas the level of $\mathrm{p}$-Smad2 was significantly reduced in the mutant myo-
statin-Tg mice. In sharp contrast, there was a significant increase in the levels of $\mathrm{p}$-Smad 2 in the mutant caveolin-3-Tg mouse muscle. In the double-Tg mice, the level of p-Smad 2 was significantly reduced compared with that in the caveolin-3-Tg mice and was similar to that in the wild-type mice. These results suggest that enhanced myostatin signaling resulting from a loss of caveolin-3 is suppressed by myostatin inhibition in the double-Tg mice.

Myostatin suppresses muscle cell growth via transcriptional regulation of the cyclin-dependent kinase (CDK) inhibitor p21 (22). Therefore we further investigated the expression profile of $p 21$ in skeletal muscle ( $n=5$ per genotype) by Northern blotting and densitometric analysis (Figure 5C). As expected, the level of $p 21 \mathrm{mRNA}$ was significantly reduced in the mutant myostatin-Tg mice. In contrast, the level of $p 21$ transcripts was significantly increased in mutant caveolin-3-Tg mice. In the double-Tg mice, the level of $p 21$ expression was significantly reduced compared with that in the caveolin-3-Tg mice and was comparable to that in wild-type mice. 

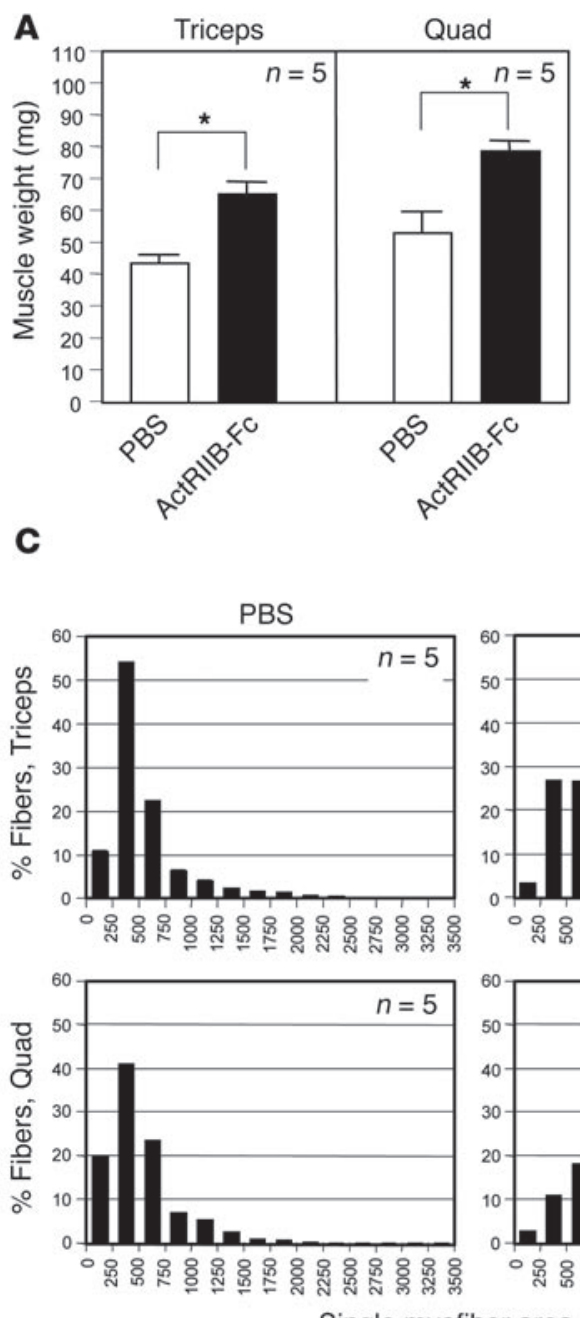

Single myofiber area $\left(\mu \mathrm{m}^{2}\right)$
B
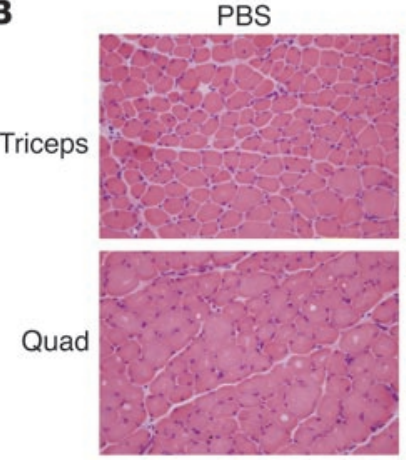

D

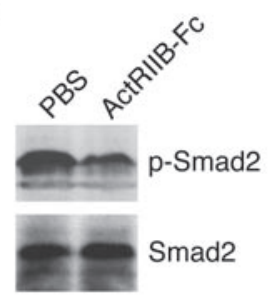

ActRIIB-Fc
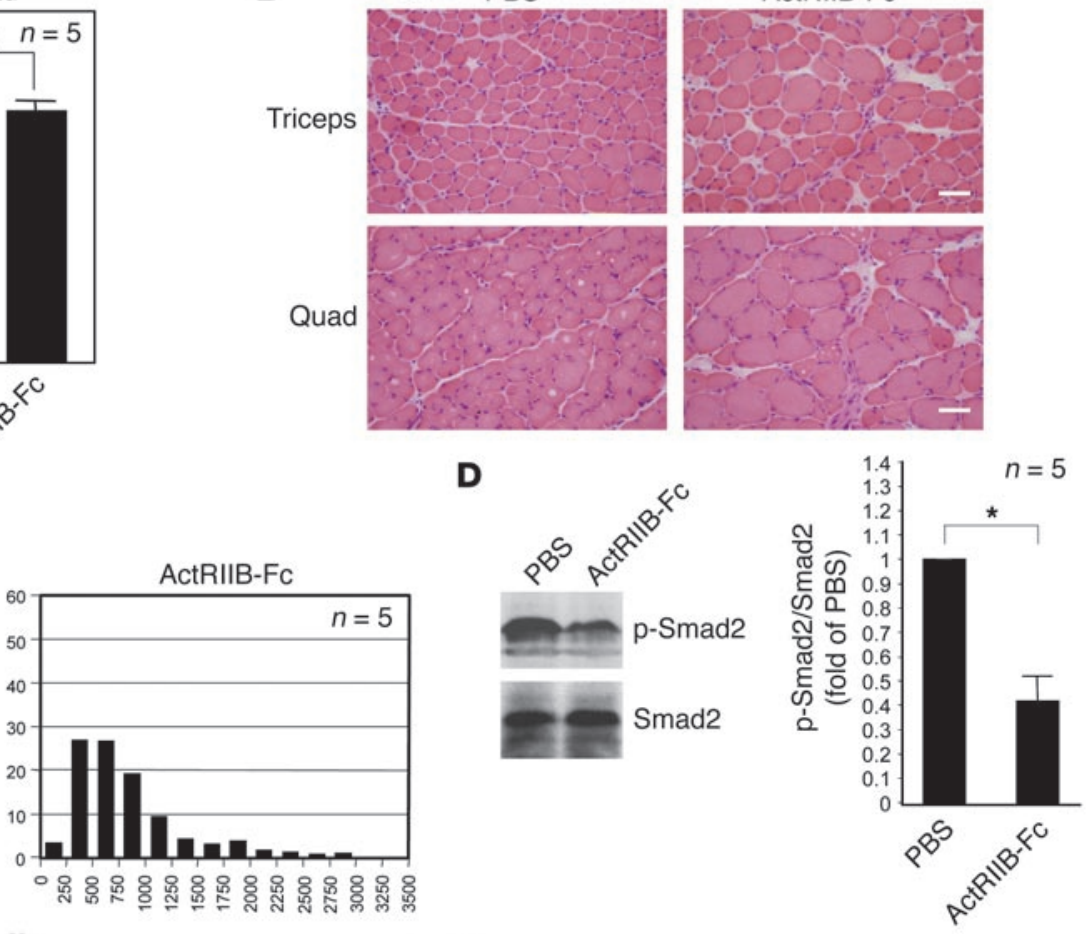

E

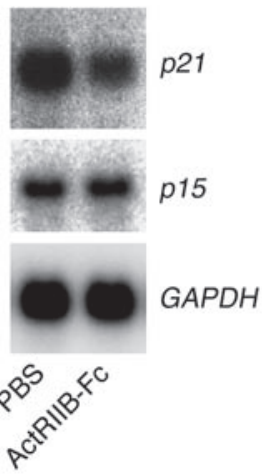

Figure 6

Intraperitoneal injection of the ActRIIB-Fc fusion protein ameliorates muscular atrophy of caveolin-3 deficiency with inhibition of myostatin signaling. (A) Comparison of muscle weight (mg) between mutant caveolin-3-Tg mice injected with ActRIIB-Fc and those injected with PBS on day 18. Triceps, triceps brachii muscle; quad, quadriceps femoris muscle. Data are expressed as mean $\pm \mathrm{SD}(n=5)$. ${ }^{*} P<0.05$. (B) H\&E staining of triceps and quadriceps muscles from mice injected with ActRIIB-Fc or PBS. Scale bar: $50 \mu \mathrm{m}$. (C) Distribution of single myofiber area of triceps and quadriceps muscles on day 18 from mice injected with ActRIIB-Fc or PBS ( $n=5 ; 250$ myofibers measured for each mouse). (D) Immunoblot analysis of total Smad2 and p-Smad2 in crude muscle homogenates from mice injected with ActRIIB-Fc or PBS (left). Quantification of p-Smad2/ Smad2 ratio by densitometric analysis (right). Values are represented as fold increase with respect to PBS-injected mice. Data are expressed as mean \pm SD $(n=5) .{ }^{*} P<0.05$. (E) Northern blot analysis in muscles from mice injected with ActRIIB-Fc or PBS for $p 21$ and $p 15$ (left). Quantification of the $p 21 / G A P D H$ ratio by densitometric analysis (right). Values are represented as fold increase with respect to PBS-injected mice. Data are expressed as mean $\pm \operatorname{SD}(n=5) .{ }^{*} P<0.05$.

Also, there was no change in the mRNA level of $p 15$, a myostatinindependent CDK inhibitor (22) (Figure 5C). Increased transcription of $p 21$ caused by a loss of caveolin-3 was reversed by myostatin inhibition in the double-Tg mice. Thus, these data suggest that myostatin inhibition suppresses enhanced intracellular myostatin signaling in caveolin-3-deficient muscle.

Intraperitoneal injection of the soluble type II myostatin receptor ameliorates muscular atrophy in mutant caveolin-3-Tg mice. To investigate the therapeutic potential of myostatin inhibition for the future treatment of LGMD1C patients, the soluble ActRIIB-Fc fusion protein, which can inhibit myostatin-ActRIIB binding $(19,24)$, was intraperitoneally injected into the mutant caveolin-3-Tg mice $(n=5)$. Injection of ActRIIB-Fc protein significantly increased the weight of triceps and quadriceps femoris muscles when compared with the control injection of PBS. H\&E staining of triceps and quadriceps femoris muscle showed increased myofiber size in the ActRIIB-Fc-injected mice (Figure 6B). Since postnatal growth of skeletal muscle occurs by myofiber hypertrophy 
(28), we examined the distribution of single myofiber area in these muscles. Compared with the PBS-injected mice, ActRIIB-Fcinjected mice showed a significant increase in single myofiber area in triceps brachii muscle $\left(507.3 \pm 204.1\right.$ versus $830.6 \pm 304.4 \mu \mathrm{m}^{2}$; $P<0.05 ; n=5)$ and quadriceps femoris muscle (642.3 \pm 234.2 versus 1067.9.4 $\pm 441.4 \mu \mathrm{m}^{2} ; P<0.05 ; n=5$; Figure $6 \mathrm{C}$ ). As expected, the levels of $\mathrm{p}-\mathrm{Smad} 2$ and $p 21$ transcripts were significantly decreased in the ActRIIB-Fc-injected mice compared with those in the PBS-injected mice (Figure 6, D and E). Thus, postnatal myostatin inhibition also reverses muscular atrophy in the caveolin-3-deficient mice in association with decreased Smad2 phosphorylation and decreased p21 expression.

\section{Discussion}

Identification of the molecular mechanism leading to muscular atrophy in the caveolin-3-deficient muscle has remained elusive (4-7). Caveolin-3 plays important roles in muscle cell signal transduction by acting as a scaffolding protein (1-3); however, the signal crosstalk between caveolin- 3 and TGF- $\beta$ superfamily members, including myostatin, has not been previously described. In the present study, we investigated molecular and functional interaction between caveolin- 3 and myostatin to better understand the pathogenesis of muscular atrophy in caveolin-3 deficiency and also tested the therapeutic potential of myostatin inhibition for the treatment of caveolin-3 deficiency.

We found that caveolin-3 colocalizes and coimmunoprecipitates with ALK4 or ALK5 type I myostatin receptor in transfected COS-7 cells and that it inhibits activation of ALK4 and ALK5 in vitro. Caveolins bind to and regulate several signaling molecules via the scaffolding domain $(2,3)$. Caveolin-binding proteins commonly possess specific caveolin-binding motifs ( $\phi \mathrm{X} \phi \mathrm{XXXX} \phi$, $\phi X X X X \phi X X \phi$, and $\phi \mathrm{X} \phi \mathrm{XXXX} \phi \mathrm{XX} \phi$, where $\phi$ indicates an aromatic or aromatic-like amino acid) (3). We examined the amino acid sequences of ALK4 and ALK5 (29) and found that there are putative caveolin-3-binding motifs in the cytoplasmic protein kinase domains of ALK4프 $\left({ }^{331} \underline{I} A H R D \underline{L} K S K N \underline{I L V K K}{ }^{345},{ }^{390} \underline{I N M K H F D S F-}\right.$ $\mathrm{KCADIY}^{404}$, and $\left.{ }^{426} \underline{Y Q L P Y Y D L V}^{436}\right)$ and ALK5 ( ${ }^{329} \underline{I A H R D L K}^{-}$ SKNILVKK ${ }^{343},{ }^{388} \underline{I N M K E E S F K R A D I Y}{ }^{402}$, and ${ }^{424} \underline{Y Q L P Y Y D L V} \underline{L}^{432}$, where underlines indicate aromatic or aromatic-like amino acids). Thus, the inhibition of the type I myostatin receptor by caveolin-3 may be due to a direct interaction between the caveolin-3 scaffolding domain and caveolin-3-binding motifs in the kinase domain of type I myostatin receptors. Alternatively, caveolin-3 may facilitate raft/caveolae-mediated endocytic degradation of ligand-bound myostatin receptors because the degradation of ligand-bound TGF- $\beta 1$ receptors is mediated by caveolin-1-coated endocytic vesicles and is accelerated by caveolin- $1(30,31)$. Further studies are needed to clarify the mechanism of how caveolin-3 inhibits activation of type I myostatin receptor.

We also demonstrated that wild-type caveolin-3 inhibits downstream intracellular events in myostatin signaling, namely phosphorylation of an effector, Smad2, and transcription of a Smad-responsive reporter gene in vitro. Other groups have demonstrated that the phosphorylation of Smad2 is mediated by activation of ALK4 or ALK5 $(20,21)$. Taken together, our results indicate that caveolin-3 negatively regulates Smad2 phosphorylation and subsequent transcription by inhibiting activation of type I myostatin receptors. Interestingly, in contrast to wild-type caveolin-3, P104L mutant caveolin-3 enhances autophosphorylation of type I receptor, subsequent Smad2 phosphorylation, and downstream transcriptional activity. Thus, the mutant caveolin-3 molecule could have a toxic gain of function.

We found that both hypoplasia and hypotrophy contribute to skeletal muscle atrophy in the mutant caveolin-3-Tg mice. Previous studies in $m d x$ mice, a mouse model of muscular dystrophy caused by dystrophin deficiency, demonstrated that the myofiber number in atrophic muscles was not decreased compared with skeletal muscles from control mice $(32,33)$. Thus, both muscle hypoplasia and hypotrophy are characteristic of caveolin-3 deficiency. In contrast, the mutant myostatin-Tg mice showed an opposite effect on myofibers (i.e., both hyperplasia and hypertrophy). These opposite effects of caveolin-3 deficiency and myostatin inhibition on myofibers suggested that caveolin-3 interacts with myostatin signaling pathways in muscle cells in vivo.

Therefore, to investigate the in vivo interaction of caveolin-3 and myostatin signaling, we mated P104L mutant caveolin-3-Tg mice with mutant myostatin-Tg mice overexpressing the myostatin prodomain to generate the double-Tg mice with both a caveolin-3 deficiency and myostatin inhibition. We found that myostatin inhibition in the double-Tg mice reversed the skeletal muscle atrophy and weakness observed in the mutant caveolin-3-Tg mice. A morphometric study demonstrated that both hypoplasia and hypotrophy seen in the skeletal muscle of mutant caveolin-3-Tg mice was reversed in the double-Tg mice. After confirming that an excessive amount of prodomain bound to endogenous $\mathrm{C}$ terminal myostatin in the mutant myostatin-Tg mice and double-Tg mice in vivo, we further investigated intracellular myostatin signaling in mouse skeletal muscles to clarify the mechanism by which myostatin inhibition rescues muscle atrophy in caveolin-3-deficient mice. Skeletal muscle from mutant myostatin-Tg mice showed a decrease in $\mathrm{p}-\mathrm{Smad} 2$ protein and downregulation of $p 21$, a myostatin-dependent CDK inhibitor (22). These results agree with previous in vitro studies showing that myostatin induces Smad2 phosphorylation and increases $p 21$ transcription (20-22) and an in vivo study demonstrating that overexpression of myostatin enhances the expression of $p 21$ in mouse skeletal muscle (9). Notably, we found that skeletal muscle from the mutant caveolin-3- $\mathrm{Tg}$ mice increased $\mathrm{p}-\mathrm{Smad} 2$ protein and upregulated $\mathrm{p} 21$, which is consistent with our in vitro results. Importantly, the double-Tg mice showed levels of p-Smad 2 and $p 21$ transcription in the skeletal muscle comparable to those of the wild-type mice, even though they were still deficient in caveolin-3. Consistent with the results from the double-Tg mice, we found that intraperitoneal injection of the soluble type II myostatin receptor ActRIIB-Fc (24) into the mutant caveolin-3-Tg mice also ameliorated muscle atrophy in association with decreased $\mathrm{p}-\mathrm{Smad} 2$ and $p 21$. These in vivo results suggest that overactivation of the intracellular myostatin signal resulting from loss of caveolin-3 plays a significant role in skeletal muscle atrophy in the LGMD1C model mice. In the current study, however, we cannot conclude that hyperphosphorylation of Smad2 and upregulated $p 21$ expression in caveolin-3-deficient skeletal muscle result simply from overactivation of myostatin signaling, since myostatin prodomain or soluble type II myostatin receptor may suppress not only myostatin but also other TGF- $\beta$ ligands, including growth and differentiation factor 11 (GDF11) (13, 19, $24,34,35)$. In fact, injection of the soluble type II myostatin receptor further increased skeletal muscle mass of muscular myostatin knockout mice (24). Thus, TGF- $\beta$ ligands other than myostatin also could be involved in the pathogenesis of muscular atrophy of caveolin-3 deficiency via Smad2-p21-mediated pathway. 
Consistent with our results, Sotgia et al. recently reported that mammary epithelial cells derived from caveolin-1 null mice showed constitutively active TGF- $\beta$ signaling, including epithelial mesenchymal transition and hyperphosphorylation of Smad2 (36). This report, together with their previous results (23) and our current results, indicates that caveolins may suppress TGF- $\beta$ superfamily-mediated growth inhibition signaling in the isotypeand cell type-specific manner: specifically, these data suggest that caveolin-1 suppresses TGF- $\beta 1$-mediated signaling in nonmuscle cells while caveolin-3 suppresses myostatin activity in muscle cells. Our current in vitro and in vivo data suggest a novel intracellular regulatory mechanism of myostatin by caveolin-3 that prevents myostatin-induced skeletal muscle atrophy.

Myostatin inhibition therapy has recently been considered for the treatment of muscular dystrophy (37-39). Intraperitoneal administration of a $\mathrm{mAb}$ against myostatin or the myostatin prodomain improves the phenotype of $m d x$ mice $(37,38)$; however, the precise molecular mechanism by which myostatin inhibition improves the phenotype of dystrophin-deficient skeletal muscle is not fully understood, and, to our knowledge, an interaction between myostatin and the dystrophin-glycoprotein complex has not been previously reported. Moreover, myostatin inhibition therapy does not improve the phenotype but rather increases the postnatal lethality of $d y$ mice, a mouse model of muscular dystrophy with laminin $\alpha 2$ deficiency (39). Therefore, myostatin inhibition therapy does not appear to be effective for all types of muscular dystrophy. It will be necessary to determine to which types of muscular dystrophy myostatin inhibition therapy could be applied and what the rescue mechanism of myostatin inhibition for muscular dystrophy is. Our present data suggest that myostatin inhibition may be a reasonable and promising therapy for caveolin-3-deficient muscular dystrophy associated with enhanced myostatin signaling. Indeed, myostatin inhibition is likely to be more successful in LGMD1C patients than in those with other types of muscular dystrophy.

\section{Methods}

Plasmid constructs. Total RNA (1 $\mu \mathrm{g})$ from mouse or human skeletal muscle was reverse-transcribed with oligo $(\mathrm{dT})_{12-18}$ primer and then subjected to PCR using the following primer sets: caveolin-3, 5'-CGGGATCCATGATGACCGAAGAGCACAC-3' (BamHI-CAV-3F, forward) and 5'-GGAATTCTTAGCCTTCCCTTCGCAGCAC-3' (EcoRI-CAV-3R, reverse); caveolin-3 with C terminal FLAG-tag, BamHI-CAV-3F and 5'-GGAATTCTTACTTATCATCATCATCCTTGTAGTCGCCTTCCCTTCGCAGCAC-3' (EcoRI-FLAGCAV-3R, reverse); caveolin-3 with C terminal HA-tag, BamHI-CAV-3F and $5^{\prime}$-GGAATTCTTAGGCGTAATCGGGGACGTCATAAGGGTAGTCGCCTTCCCTTCGCAGCAC-3' (EcoRI-HA-CAV-3R, reverse); ALK4 with C terminal FLAG-tag, 5'-CCGGAATTCATGGCGGAGTCGGCCGGAGCC-3 (EcoRI-ALK4F, forward) and 5'-CCGCTCGAGCTACTTATCATCATCATCCTTGTAGTCGATCTTCACGTCTTCCTGCACG-3' (XhoI-FLAG-ALK4R, reverse); and ALK5 with an N terminal FLAG-tag, 5'-CCATCGATATGGAGGCGGCGGTCGCTGCTC-3' (ClaI-ALK5F, forward) and $5^{\prime}$-TGGCTGCAGTTACTTATCATCATCATCCTTGTAGTCCATTTTGATGCCTTCCTGTTG-3' (PstI-FLAG-ALK5R, reverse). The PCR products were subcloned into the pCS2+ vector (40) using the appropriate restriction sites. Using the QuickChange Site-Directed Mutagenesis Kit (Stratagene), the P104L mutation of caveolin-3 cDNA was introduced into pCS2+wild-type caveolin-3 vector or pCS2+C terminal FLAG-tagged wildtype caveolin-3 vector with the following primers: 5'-CACATCTGGGCCGTGGTGCTCTGCATTAAGAGCTACCTG-3' (P104L-CAV-3F, forward) and $5^{\prime}$-CAGGTAGCTCTTAATGCAGAGCACCACGGCCCAGATGTG-3'
(P104L-CAV-3R, reverse). HA-tagged ALK4, HA-tagged constitutively active ALK4, HA-tagged ALK5, and HA-tagged constitutively active ALK5 were constructed and provided by K. Miyazono (University of Tokyo, Tokyo, Japan) and T. Imamura (The Cancer Institute of the Japanese Foundation for Cancer Research) as described previously $(41,42)$. The pGL3-(CAGA) ${ }_{12-}$ luciferase reporter containing the Smad-binding sequence (CAGA) was provided by C.-H. Heldin (Uppsala University, Uppsala, Sweden) (24). The pCMV- $\beta$-gal vector was used as described previously (43).

Cell culture, transfection, and induction. HEK293 human embryonic kidney and COS-7 monkey kidney cells were maintained in DMEM containing 10\% FBS, $2 \mathrm{mM}$ L-glutamine, $0.1 \mathrm{mM}$ nonessential amino acids, and $50 \mu \mathrm{g} / \mathrm{ml}$ kanamycin. Cells were transfected with plasmid DNA-lipid complex using the FuGENE 6 transfection reagent (Roche Diagnostics) according to the manufacturer's protocol. Recombinant myostatin (R\&D Systems) was prepared as a $100 \mu \mathrm{g} / \mathrm{ml}$ stock solution in $0.1 \%$ BSA with $4 \mathrm{mM} \mathrm{HCl}$.

Immunofluorescence and immunoprecipitation analyses of transfected cells. COS-7 cells cotransfected with FLAG-tagged caveolin-3 and HA-tagged type I receptor were fixed, permeabilized, and double-stained with anti-rabbit caveolin-3 polyclonal $\mathrm{Ab}$ (BD Bioscience) and an anti-HA mAb 12CA5 (Roche Diagnostics) for 60 minutes. After washing with PBS, the cells were incubated for 30 minutes with Alexa Fluor 488-conjugated anti-rabbit and Alexa Fluor 594-conjugated anti-mouse IgG Abs (Invitrogen). Immunofluorescent images were captured using a TCS SP2 Laser Scanning Confocal Microscope (Leica Microsystems). For immunoprecipitation, COS-7 cells cotransfected with FLAG- or HA-tagged caveolin-3 and HA- or FLAGtagged type I receptor were incubated for 30 minutes on ice with a lysis buffer containing $50 \mathrm{mM}$ Tris- $\mathrm{HCl}$ ( $\mathrm{pH} 7.4$ ), $50 \mathrm{mM} \mathrm{NaCl}, 1 \%$ Triton X-100, and $100 \mathrm{mM}$ octyl glucoside (Sigma-Aldrich) supplemented with a proteinase inhibitor cocktail (complete, EDTA-free; Roche Diagnostics). Cells were precleared by centrifugation at $10,000 \mathrm{~g}$ for 30 minutes, after which anti-FLAG mAb M2 agarose gel (Sigma-Aldrich) was added to precleared lysates. After rotary mixing for 2 hours at $4^{\circ} \mathrm{C}$, immunoprecipitates were washed 3 times with lysis buffer. The immunoprecipitates or whole cell lysates were separated by SDS-PAGE (3\% to $18 \%$ linear gradient of acrylamide), transferred to a PVDF membrane, and subjected to immunoblot analysis. The membrane was blocked with $5 \%$ milk in Tris-buffed saline (50 mM Tris- $\mathrm{HCl}[\mathrm{pH} 7.5]$ and $137 \mathrm{mM} \mathrm{NaCl}$ ) containing $0.1 \%$ Tween-20 and then incubated overnight at $4{ }^{\circ} \mathrm{C}$ with anti-FLAG mAb M2 (SigmaAldrich) or anti-HA mAb $12 \mathrm{CA} 5$. After washing with Tris-buffered saline containing $0.1 \%$ Tween- 20 , the blots were incubated with horseradishconjugated anti-mouse IgG Ab (Amersham Biosciences). Immunoreactive bands were visualized using ECL reagents (Amersham Biosciences).

In vitro autophosphorylation of constitutively active ALK4 or ALK5. Lysates from COS-7 cells cotransfected with FLAG-tagged wild-type or P104L mutant caveolin-3 and HA-tagged constitutively active forms of type I receptor, ALK4 or ALK5, were immunoprecipitated with anti-HA 12CA5 agarose gel (SigmaAldrich). Immunoprecipitates of constitutively active ALK4 or ALK5 were equilibrated with $50 \mu \mathrm{l}$ of kinase reaction buffer ( $40 \mathrm{mM}$ HEPES [pH 7.4], $10 \mathrm{mM} \mathrm{MgCl}_{2}, 3 \mathrm{mM} \mathrm{MnCl}_{2}, 10 \mathrm{mM} \beta$-glycerol-phosphate, $10 \mathrm{mM} \mathrm{NaF}$, and $2 \mathrm{mM}$ DTT) for 5 minutes on ice. The kinase reaction was initiated by adding $10 \mu \mathrm{Ci}$ of $\left[\gamma^{-32} \mathrm{P}\right] \mathrm{ATP}$. After incubation for 20 minutes at $25^{\circ} \mathrm{C}$, the reaction was terminated by addition of $50 \mu \mathrm{l}$ of $\times 2$ SDS-PAGE sample buffer and boiling for 5 minutes. The sample was subsequently subjected to SDS-PAGE ( $3 \%$ to $18 \%$ linear gradient of acrylamide), and phosphorylated constitutively active ALK4 or ALK5 was detected by autoradiography using a Fuji Imaging Plate (Fujifilm). Total type I receptor or caveolin-3 was immunoblotted using anti-HA mAb 12 CA5 or anti-FLAG mAb M2.

In vitro analysis of Smad2 phosphorylation. Recombinant myostatin was added to COS-7 cells transfected with wild-type caveolin-3, P104L mutant caveolin-3, or empty vector control. After 24 hours, cells were lysed in PBS 
containing $1 \mathrm{mM}$ EDTA and $0.1 \%$ Triton X-100, and resulting cell lysates were subjected to immunoblot analysis using a rabbit polyclonal $\mathrm{Ab}$ against Smad2 or p-Smad2 (Ser465/467; Cell Signaling Technology).

Luciferase assays for myostatin activity. HEK293 cells were seeded in 12-well plates at a density of $1.0 \times 10^{5}$ cells per well and cultured for 24 hours. Cells were then cotransfected with the pGL3-(CAGA) ${ }_{12}$-luciferase reporter gene, the $\mathrm{pCMV}-\beta$-gal, and either empty vector ( $\mathrm{pCS} 2+$ ), wild-type caveolin-3 (pCS2 + wild-type CAV-3), or P104L mutant caveolin-3 (pCS2 + mutant CAV-3). After 24 hours, medium was replaced with serum-free DMEM containing $20 \mathrm{ng} / \mathrm{ml}$ recombinant myostatin. After an additional 24 hours, cells were lysed with $\times 1$ lysis buffer (Promega), and luciferase activity in the cell lysates was determined by a luciferase reporter assay system (Promega) using an LB9506 MiniLumat Luminometer (Berthold Technologies). Luciferase activities were normalized by $\beta$-gal activity as described previously (43).

Generation of double-Tg mice coexpressing P104L mutant caveolin-3 and myostatin prodomain. We previously generated CAV-3 $3^{\text {P104L }}$ and $\operatorname{MSTN}^{\text {Pro }}$ mice $(5,12)$. Heterozygous mating of these mice gave rise to $\mathrm{F} 1$ offspring with 4 distinct genotypes: wild-type, mutant caveolin-3-Tg $\left(\mathrm{CAV}-3^{\mathrm{P} 104 \mathrm{~L}}\right)$, mutant myostatin$\operatorname{Tg}\left(\right.$ MSTN $\left.^{\text {Pro }}\right)$, and double $\mathrm{Tg}\left(\mathrm{CAV}-3^{\mathrm{P} 104 \mathrm{~L}} / \mathrm{MSTN}^{\mathrm{Pro}}\right)$. The presence of transgenes (P104L caveolin-3 or myostatin prodomain) was determined by PCR amplification from tail DNA as described previously $(5,12)$. All animal experiments were performed at the Laboratory Animal Center with the approval of the Animal Research Committee at the Kawasaki Medical School.

Computed tomographic analysis of muscle volume. Axial-computed tomography at the center of the distal hind limb in mice was performed using a LaTheta LTC-100 (Aloka).

Immunohistochemical and morphometric analyses. Unfixed distal hind limbs of mice were snap-frozen in liquid nitrogen-cooled isopentane and sectioned transversely $(10 \mu \mathrm{m})$ at the center of the TA muscle using a cryostat (Leica Microsystems) with an A35 Microtome Blade (Tech-Jam) as described previously (44). Sections were postfixed in $1 \%$ formalin in PBS for 10 minutes and then immunostained according to the M.O.M. procedure (Vector Mouse on Mouse Kit; Vector Laboratories) using a rabbit polyclonal $\mathrm{Ab}$ against caveolin-3 (BD Biosciences) or a rat $\mathrm{mAb}$ against laminin $\alpha 2$ (clone 4H8-2; Sigma-Aldrich) followed by rabbit or rat FITCconjugated secondary Abs. The single myofiber area in the TA muscle $(n=5$; 250 myofibers measured for each mouse) and the total myofiber number in the TA muscle $(n=5)$ were measured from the fluorescence images of anti-laminin $\alpha 2$-stained sections using the 2-color technique with Lumina Vision software version 2.0 (Mitani Corp.).

Grip strength and treadmill exercise tests. Mice grasping wire mesh with their forelimbs and hind limbs were pulled horizontally by their tails until they lost their grip. Peak grip strength (g) was measured using an MK-380S automated Grip Strength Meter (Muromachi). For Supplemental Video 1, the mice were placed on an MK-680S treadmill (Muromachi) with a shock bar $(1 \mathrm{~mA})$ and run at $14 \mathrm{~m} / \mathrm{min}$ for 10 minutes.

Immunoprecipitation and immunoblot analyses of skeletal muscles and sera. As described previously $(26,27)$, myostatin was immunoprecipitated from skeletal muscle or serum samples from each mouse at 10 weeks of age using an $\mathrm{Ab}$ against the myostatin $\mathrm{C}$ terminal peptide (R\&D Systems). Immunoblot analysis was performed using a polyclonal $\mathrm{Ab}$ against the myostatin prodomain (gift of Wyeth). Control recombinant myostatin prodomain was purchased from R\&D Systems. For immunoblot analysis of Smad2 and p-Smad2, skeletal muscle from 10-week-old mice was homogenized in 10 volumes (w/v) of a buffer consisting of $50 \mathrm{mM}$ Tris$\mathrm{HCl}$ (pH 7.4), $100 \mathrm{mM} \mathrm{NaCl}, 1 \mathrm{mM}$ EDTA, $5 \mathrm{mM} \beta$-mercaptoethanol, $0.1 \mathrm{mM}$ PMSF, and $1 \mathrm{mM}$ benzamidine. The crude extracts were separated by SDS-PAGE ( $3 \%$ to $18 \%$ linear gradient of acrylamide) and transferred to PVDF. Immunoblot analysis was performed using the same Abs described for in vitro analysis of Smad2 phosphorylation.
Northern blot analysis. RT-PCR products of mutant caveolin- 3 transgene (5), mutant myostatin transgene (12), mouse p21 (nt1-255), and mouse p15 (nt1-421) were subcloned into the PCRII-TOPO vector (Invitrogen) and then digested with EcoRI. The digested insert was separated by agarose gel electrophoresis and extracted using a Rapid Gel Extraction System (Marligen Bioscience). Each DNA fragment was then labeled with $\left[\alpha{ }^{32} \mathrm{P}\right] \mathrm{dCTP}$ using the MegaPrime DNA Labeling System (Amersham Biosciences). Twenty micrograms of total RNA from the skeletal muscle of a 10 -week-old mouse from each strain was separated on a $0.7 \%$ agarose gel containing $7 \%$ formaldehyde and blotted onto Hybond-N+ (Amersham Biosciences). Hybridization was performed at $42^{\circ} \mathrm{C}$ for 24 hours, and autoradiography was performed using a Fuji Imaging Plate (Fujifilm).

Intraperitoneal injection of the ActRIIB-Fc fusion protein to mutant caveolin-3-Tg mice. According to the method described previously (24), $10 \mathrm{mg} / \mathrm{kg}$ of human ActRIIB-Fc fusion protein, purchased from R\&D Systems, was injected into 6-week-old P104L mutant caveolin-3-Tg mice on days 1, 4, 8, and $15(n=5)$. Mice were sacrificed on day 18 for analysis of skeletal muscle. Morphometrical analysis of single myofiber area in the triceps brachii and quadriceps femoris muscles $(n=5 ; 250$ myofibers measured for each muscle), Smad2 phosphorylation analysis, and p21 expression analysis were performed using the same method as described above.

Statistics. Statistical analysis was performed on paired observations using Bonferroni's test after 1-way ANOVA. $P$ values less than 0.05 were considered significant.

\section{Acknowledgments}

We wish to thank Wyeth and C.-H. Heldin (Uppsala University), K. Miyazono (Department of Molecular Pathology, University of Tokyo), and T. Imamura (Department of Biochemistry, The Cancer Institute of the Japanese Foundation for Cancer Research) for reagents and A.C. McPherron (National Institute of Diabetes and Digestive and Kidney Diseases, NIH) for comments on the manuscript. We are grateful to $\mathrm{N}$. Akazawa for critical reading of the manuscript. We also thank T. Murakami (Division for Therapies against Intractable Diseases, Institute for Comprehensive Medical Science, Fujita Health University), S.-i. Nishimatsu, and T. Nohno (Department of Molecular Biology, Kawasaki Medical School) as well as S. Hirano, H. Kitao, and M. Takata (Department of Immunology, Kawasaki Medical School) for valuable advice, and we thank M. Kita (Laboratory Animal Center, Kawasaki Medical School), K. Yamane, K. Uehira (Electron Microscopy Center, Kawasaki Medical School), N. Naoe, T. Kawase, and Y. Kuroda (Division of Neurology, Kawasaki Medical School) for technical assistance. This work was supported by a research grant for Nervous and Mental Disorders from the Ministry of Health, Labor and Welfare (14B-4); grants for research on Psychiatric and Neurological Diseases and Mental Health from the Ministry of Health, Labor and Welfare of Japan (15131301 and 17231401) and from the Japan Society for the Promotion of Science (KAKENHI 14370212); and by research project grants from Kawasaki Medical School (15-115B, 16-601, and 17-605S).

Received for publication March 14, 2006, and accepted in revised form July 11, 2006.

Address correspondence to: Yoshihide Sunada, Division of Neurology, Department of Internal Medicine, Kawasaki Medical School, 577 Matsushima, Kurashiki, Okayama 701-0192, Japan. Phone: 81-86-462-1111; Fax: 81-86-462-1199; E-mail: ysunada@med. kawasaki-m.ac.jp. 
1. Parton, R.G. 2003. Caveolae-from ultrastructure to molecular mechanisms. Nat. Rev. Mol. Cell Biol. 4:162-167.

2. Galbiati, F., Razani, B., and Lisanti, M.P. 2001. Emerging themes in lipid rafts and caveolae. Cell. 106:403-411.

3. Couet, J., Li, S., Okamoto, T., Ikezu, T., and Lisanti, M.P. 1997. Identification of peptide and protein ligands for the caveolin-scaffolding domain. Implications for the interaction of caveolin with caveolaeassociated proteins. J. Biol. Chem. 272:6525-6533.

4. Minetti, C., et al. 1998. Mutations in the caveolin-3 gene cause autosomal dominant limb-girdle muscular dystrophy. Nat. Genet. 18:365-368.

5. Sunada, Y., et al. 2001. Transgenic mice expressing mutant caveolin-3 show severe myopathy associated with increased nNOS activity. Hum. Mol. Genet. 10:173-178

6. Smythe, G.M., Eby, J.C., Disatnik, M.H., and Rando, T.M. 2003. A caveolin-3 mutant that causes limb girdle muscular dystrophy type $1 \mathrm{C}$ disrupts Src localization and activity and induces apoptosis in skeletal myocytes. J. Cell Sci. 116:4739-4749.

7. Hernandez-Deviez, D.J., et al. 2006. Aberrant dysferlin trafficking in cells lacking caveolin or expressing dystrophy mutants of caveolin-3. Hum. Mol. Genet. 15:129-142.

8. McPherron, A.C., Lawler, A.M., and Lee, S.-J. 1997. Regulation of skeletal muscle mass in mice by a new TGF-beta superfamily member. Nature. 387:83-90.

9. Zimmers, T.A., et al. 2002. Induction of cachexia in mice by systemically administered myostatin. Science. 296:1486-1488.

10. Reisz-Porszasz, S., et al. 2003. Lower skeletal muscle mass in male transgenic mice with muscle-specific overexpression of myostatin. Am.J. Physiol. Endocrinol. Metab. 285:E876-E888.

11. Grobet, L., et al. 2003. Modulating skeletal muscle mass by postnatal, muscle-specific inactivation of the myostatin gene. Genesis. 35:227-238.

12. Nishi, M., et al. 2002. A missense mutant myostatin causes hyperplasia without hypertrophy in the mouse muscle. Biochem. Biophys. Res. Commun. 293:247-251.

13. Thies, R.S., et al. 2001. GDF-8 propeptide binds to GDF- 8 and antagonizes biological activity by inhibiting GDF-8 receptor binding. Growth Factors. 18:251-259

14. Wolfman, N.M., et al. 2003. Activation of latent myostatin by the BMP-1/tolloid family of metalloproteinases. Proc. Natl. Acad. Sci. U. S. A. 100:15842-15846.

15. Yang, J., et al. 2001. Expression of myostatin pro domain results in muscular transgenic mice. Mol. Reprod. Dev. 60:351-361.

16. Pirottin, D., et al. 2005. Transgenic engineering of male-specific muscular hypertrophy. Proc. Natl. Acad. Sci. U. S. A. 102:6413-6418.

17. Yang, J., and Zhao, B. 2006. Postnatal expression of myostatin propeptide cDNA maintained high muscle growth and normal adipose tissue mass in transgenic mice fed a high-fat diet. Mol. Reprod. Dev. 73:462-469.

18. Massague, J. 1998. TGF-beta signal transduction. Annu. Rev. Biochem. 67:753-791.

19. Lee, S.-J., and McPherron, A.C. 2001. Regulation of myostatin activity and muscle growth. Proc. Natl. Acad. Sci. U. S. A. 98:9306-9311.

20. Rebbapragada, A., Benchabane, H., Wrana, J.L., Celeste, A.J., and Attisano, L. 2003. Myostatin signals through a transforming growth factor betalike signaling pathway to block adipogenesis. Mol. Cell. Biol. 23:7230-7242.

21. Rios, R., Fernandez-Nocelos, S., Carneiro, I., Arce, V.M., and Devesa, J. 2004. Differential response to exogenous and endogenous myostatin in myoblasts suggests that myostatin acts as an autocrine factor in vivo. Endocrinology. 145:2795-2803.

22. Thomas, M., et al. 2000. Myostatin, a negative regulator of muscle growth, functions by inhibiting myoblast proliferation. J. Biol. Chem. 275:40235-40243.

23. Razani, B., et al. 2001. Caveolin-1 regulates transforming growth factor (TGF)-beta/SMAD signaling through an interaction with the TGF-beta type I receptor. J. Biol. Chem. 276:6727-6738.

24. Lee, S.-J., et al. 2005. Regulation of muscle growth by multiple ligands signaling through activin type II receptors. Proc. Natl. Acad. Sci. U. S. A. 102:18117-18122.

25. Jonk, L.J., Itoh, S., Heldin, C.-H., ten Dijke, P., and Kruijer, W. 1998. Identification and functional characterization of a Smad binding elements (SBE) in the JunB promoter that acts as a transforming growth factor-beta, activin, and bone morphogenetic protein-inducible enhancer. J. Biol. Chem. 273:21145-21152.

26. Hill, J.J., et al. 2002. The myostatin propeptide and the follistatin-related gene are inhibitory binding proteins of myostatin in normal serum. J. Biol. Chem. 277:40735-40741.

27. Schuelke, M., et al. 2004. Myostatin mutation associated with gross muscle hypertrophy in a child. N. Engl. J. Med. 350:2682-2688.

28. Campion, D.R. 1984. The muscle satellite cell: a review. Int. Rev. Cytol. 87:225-251.

29. Feng, X.-H., and Derynck, R.A. 1997. A kinase subdomain of transforming growth factor-beta (TGF-beta) type I receptor determines the TGF-beta intracellular signaling specificity. EMBO J. 16:3912-3923.

30. Di Guglielmo, G.M., Le Roy, C., Goodfellow, A.F., and Wrana, J.L. 2003. Distinct endocytic pathways regulate TGF-beta receptor signalling and turnover. Nat. Cell Biol. 5:410-421.

31. Le Roy, C., and Wrana, J.L. 2005. Clathrin- and non-clathrin-mediated endocytic regulation of cell signalling. Nat. Rev. Mol. Cell Biol. 6:112-126.

32. DiMario, J.X., Uzman, A., and Strohman, R.C. 1991. Fiber regeneration is not persistent in dystrophic (MDX) mouse skeletal muscle. Dev. Biol. 148:314-321.

33. Pastoret, C., and Sebille, A. 1993. Further aspects of muscular dystrophy in mdx mice. Neuromuscul. Disord. 3:471-475.

34. Oh, S.P., et al. 2002. Activin type IIA and IIB receptors mediate Gdf11 signaling in axial vertebral patterning. Genes Dev. 16:2749-2754.

35. De Caestecker, M. 2004. The transforming growth factor-beta superfamily of receptors. Cytokine Growth Factor Rev. 15:1-11.

36. Sotgia, F., et al. 2006. Caveolin-1 deficiency (-/-) conveys premalignant alterations in mammary epithelia, with abnormal lumen formation, growth factor independence, and cell invasiveness. Am. J. Pathol. 168:292-309.

37. Bogdanovich, S., et al. 2002. Functional improvement of dystrophic muscle by myostatin blockade. Nature. 420:418-421.

38. Bogdanovich, S., Perkins, K.J., Krag, T.O.B., Whittemore, L.-A., and Khurana, T.S. 2005. Myostatin propeptide-mediated amelioration of dystrophic pathology. FASEB J. 19:543-549.

39. Li, Z.F., Shelton, G.D., and Enovall, E. 2005. Elimination of myostatin does not combat muscular dystrophy in dy mice but increase postnatal lethality. Am. J. Pathol. 166:491-495.

40. Narita, T., et al. 2005. Wnt10a is involved in AER formation during chick limb development. Dev. Dyn. 233:282-287.

41. Kawabata, M., Inoue, H., Hanyu, A., Imamura, T., and Miyazono, K. 1998. Smad proteins exist as monomers in vivo and undergo homo- and heterooligomerization upon activation by serine/threonine kinase receptors. EMBO J. 17:4056-4065.

42. Murakami, G., Watabe, T., Takaoka, K., Miyazono, K., and Imamura, T. 2003. Cooperative inhibition of bone morphogenetic protein signaling by Smurf1 and inhibitory Smads. Mol. Biol. Cell. 14:2809-2817.

43. Funaba, M., et al. 2003. Transcriptional activation of mouse mast cell Protease- 7 by activin and transforming growth factor-beta is inhibited by microphthalmia-associated transcription factor. J. Biol. Chem. 278:52032-52041.

44. Sasaoka, T., et al. 2003. Pathological analysis of muscle hypertrophy and degeneration in muscular dystrophy in gamma-sarcoglycan-deficient mice. Neuromuscul. Disord. 13:193-206. 